\title{
Q fever in the Netherlands - what matters is SERIOUSNESS OF DISEASE RATHER THAN QUANTITY
}

\author{
G Cilla ${ }^{1,2}$, M Montes $^{1,2}$, E. Pérez-Trallero (mikrobiol@terra.es)1,2,3 \\ 1. Servicio de Microbiología, Hospital Donostia, San Sebastián, Spain \\ 2. Centro de Investigación Biomédica en Red Enfermedades Respiratorias CIBERes, San Sebastián, Spain \\ 3. Departamento de Medicina Preventiva y Salud Pública, Facultad de Medicina, Universidad del País Vasco, San Sebastián, Spain
}

To the editor: We have read with interest the article by Schimmer $B$ et al. [1] about $Q$ fever in the Netherlands, an outbreak that captured the attention of the mass media and was labelled as "unique in the world". We wish to contribute our experience regarding the clinical manifestations and potential seriousness of this disease and make some comments about the difficulties of diagnosis encountered in endemic areas.

Between 1984 and 2004, as a result of routine clinical diagnoses and without active surveillance, 1,261 cases of $Q$ fever were detected in our region (Gipuzkoa, Basque country, north of Spain, 690,000 inhabitants) [2]. The annual number of cases ranged from 19 to 153 , with two major outbreaks, at the beginning and at the end of the 1990s. The Gipuzkoa experience showed several similarities with that of the south of the Netherlands, both regions having a temperate oceanic climate and generally abundant rainfall spread out over the year. The most common clinical manifestation of acute $Q$ fever in our report was atypical pneumonia (79\%), with hepatitis being very infrequently found. Our study also revealed that most cases occurred in men $(75 \%)$, in adults $(70 \%$ between 25 and 54 years) and between March and June (64.3\%), a period that coincides with the greatest parity of sheep and goats and the season when people frequently take walks in the countryside. There were no deaths, fewer than $2 \%$ of the patients required admission to the intensive care unit, and only two cases of chronic $Q$ fever (two patients with prosthetic valve endocarditis) were detected. In regions with the same climate, the seriousness of the disease and clinical manifestations are similar while differing from those in other climatological regions, suggesting that different Coxiella burnetii types circulate in regions with different climates.

To date, diagnosis of $Q$ fever relies on serology, immunofluorescence (IF) being the reference technique [3]. The diagnostic criteria used by us are more restrictive than those usually employed. For acute $Q$ fever, using the IF, C. burnetii phase II, Bio-Mèrieux, these criteria were as follows: seroconversion of IgG and/or IgM (from negative to $\geq 1 / 128$ ), a four-fold or greater increase in IgG titre in two paired sera, or IgM titre $\geq 1 / 256$ in a single sample after removing rheumatic factor. The Tissot-Dupont cut-off for acute $Q$ fever (anti-phase // IgG titre $\geq 1 / 200$ and anti-phase II IgM titre $\geq 1 / 50$ ) [3] would not be useful in our area, since many patients without acute $Q$ fever would meet these criteria.

We reviewed the presence of these lower titres among the serological results obtained in two years: one year with an outbreak
(1992: 153 cases) and another without outbreaks (2002: 43 cases). If an IgM titre of 1:50 with IgG titre $\geq 1 / 200$ had been used as the diagnostic criteria, the number of acute cases would have increased by $22.2 \%$ (187 cases instead of 153 ) and $39.5 \%$ (60 instead of 43) in 1992 and 2002, respectively. These patients were excluded after we ascertained that they showed no clinical manifestations of infection. Moreover, we detected IgM titres $\geq 1 / 64$ with IgG $\geq 1 / 256$ in a further 46 patients without chronic infection, who had already been diagnosed with acute $Q$ fever five or more months previously. In 24 of these patients, high antibody titres persisted for over one year.

As the number of detected cases may be closely related to the intensity of the search and the methods and criteria used in the diagnosis, we believe that exhaustive surveillance under pressure from the media could lead to over-diagnosis and unnecessary treatments which, like that of pregnant women, are not free of risk.

\section{References}

1. Schimmer B, Morroy G, Dijkstra F, Schneeberger PM, Weers-Pothoff G, Timen A, Wijkmans $C$, van der Hoek W. Large ongoing $Q$ fever outbreak in the south of The Netherlands, 2008. Euro Surveill. 2008;13(31):pii=18939. Available from: http://www.eurosurveillance.org/ViewArticle.aspx?ArticleId=18939

2. Montes M., Cilla G., Vicente D., Ercibengoa M., Perez-Trallero E. Gipuzkoa, Basque Country, Spain (1984-2004), a Hyperendemic Area of Q Fever. Ann N Y Acad Sci. 2006;1078:129-132.

3. Maurin M, Raoult D. Q fever. Clin Microbiol Rev. 1999;12:518-53.

This article was published on 11 September 2008.

Citation style for this article: Cilla G, Montes M, Pérez-Trallero E. Q fever in the Netherlands - what matters is seriousness of disease rather than quantity. Euro Surveill. 2008;13(37):pii=18975. Available online: http://www.eurosurveillance.org/ ViewArticle.aspx?ArticleId=18975 